\title{
IDIOPATHIC PULMONARY HAEMOSIDEROSIS
}

\author{
BY \\ G. B. TAIT AND M. CORRIDAN \\ From the General Hospital, Dewsbury
}

(RECEIVED FOR PUbLICATION APRIL 27, 1952)

Idiopathic pulmonary haemosiderosis (essential brown induration of the lungs) is now well recognized in children. Originally described by Ceelen in 1931, many other authors have added cases (Wyllie, Sheldon, Bodian, and Barlow, 1948).

It manifests itself clinically by attacks of tachycardia, pallor, anaemia, cyanosis, haemoptysis, and sometimes jaundice, together with clinical or radiological signs in the chest. The earlier attacks subside and are often mistaken for haemolytic episodes. Pathologically there is haemorrhagic pulmonary consolidation. The alveoli are filled with" haemosiderin-laden phagocytes (" heart failure cells") and these are also present in the alveolar walls.

A similar condition has been described only three times in adults. Belfrage and Waldenstrom described a case which developed at the age of 16 years and ended fatally at 19 years (Wyllie and others, 1948). Walton and Williams (1951) described another case in the same age group. Only one patient whose symptoms started later in life has been described previously, a man aged 38 years, by Borsos-Nachtnebel (Scheidegger and Dreyfus, 1945). We describe here a case occurring in a man aged 31 years.

\section{Clinical Features}

The patient was a school-teacher aged 31 . He had enjoyed good health until two and a half years previously, when he began to suffer from occasional bouts of diarrhoea, sometimes severe but without mucus or blood. He had been investigated fully for this complaint at another hospital, but no evidence of organic disease had been found.

$\mathrm{He}$ attended the out-patient department of the General Hospital, Dewsbury, on January 17, 1951, with a history of one week's cough with sputum which had been blood-stained. The blood-staining had ceased at the time of attendance. Otherwise he felt well, but had lost some weight over the past three years. There was nothing relevant in the family history. Physical examination revealed a spare individual of average height. The respiratory and other systems were normal. There was no telangiectasis of the skin. His blood pressure was 110/90. Blood examination showed haemoglobin $109 \%$ (16.1 g. pef $100 \mathrm{ml}$.), erythrocyte sedimentation rate (E.S.R W $1 \mathrm{~mm}$. fall in one hour. A radiograph of the chest showed no abnormality, and the sputum did not cons tain tubercle bacilli. Examination of stools revealed no evidence of parasites.

The patient was kept under observation as an ou疍 patient. Repeated physical and radiological exam 5 nations were negative. Bronchoscopy on May $1 \Phi$ 1951 , by Mr. G. H. Wooler showed nothing to account for the bleeding.

From time to time bouts of haemoptysis recurreq $\mathrm{He}$ was admitted to hospital on August 12, 1956 after a profuse haemoptysis.

No clinical abnormalities were detected excep early clubbing of the fingers. The blood haemo globin was $77 \%(11.4 \mathrm{~g}$. per $100 \mathrm{ml}$.), the E.S.R was $13 \mathrm{~mm}$. in one hour; otherwise the blood w\$ normal. The stools were re-examined, with negative results; they had a normal fat content. Repeate examination of the sputum showed no acid-fast bacilli. The Wassermann and Kahn tests were nega tive. A chest radiograph revealed no abnormaliti: From time to time he complained of vague discon fort behind the sternum and attacks of feeling he might choke. He improved on bed-rest, and the bleeding gradually ceased. $\mathrm{He}$ was discharged com August 28, 1951.

He was readmitted on November 1, 1951, in 8 collapsed state after a severe haemoptysis. He wa transfused with blood, but in spite of this deterios ated and died.

The body was that of a thin, red-haired freckled man with pale skin and mucous membranes. The lungs were large and voluminous and did not collapse on opening the pleurab cavities, which were normal. The pleura coverit each lung was strikingly dark purple. The midzones were particularly firm, and haemorrhage fluid could be squeezed from the lung parenchymat. The trachea and bronchi contained a large amoun of haemorrhagic fluid. A superior bronch 8 pulmonary and an inferior tracheo-bronchi@ lymph node on the left side, and a superior arif an inferior tracheo-bronchial lymph node on the right side, showed moderate enlargement. The cut 
surface of these enlarged nodes was greyish-brown. The right side of the heart was slightly dilated but otherwise appeared normal. The liver and spleen were normal in size and appearance. The gastrointestinal tract and other abdominal organs were normal macroscopically. The pia-arachnoid was congested and the anterior part of the corpus callosum showed several purpuric spots of about 2 to $4 \mathrm{~mm}$. in diameter.

Microscopical Examination.-Many sections from various parts of both lungs were examined, and showed the same picture. Very few alveoli contained air. There were areas of recent haemorrhage, in which the alveoli were stuffed with fresh red blood cells representing the fatal haemoptysis. The number of alveoli in these haemorrhagic areas was quite variable. The older haemoptyses and intra-alveolar haemorrhage were represented by areas in which the alveoli contained numerous haemosiderin-laden phagocytes (siderophages) which gave a brilliant Prussian blue reaction (Fig. 1 ), and also by areas of casts in the alveolar ducts and related alveoli. The central part of these casts, in the ducts, was composed of red blood cells, while the alveolar or peripheral part showed siderophages and fibrin threads which stained with Mallory's phosphotungstic haematoxylin (Fig. 2). These areas of recent and old haemorrhages alternated irregularly throughout the lungs. The alveolar walls contained siderophages and free granules of haemosiderin.

There was a slight increase of the reticular fibrils of the alveolar walls. The elastic tissue of the alveoli showed neither iron impregnation nor foreign-body giant-cell reaction, and, apart from fractures, was regarded as being within normal

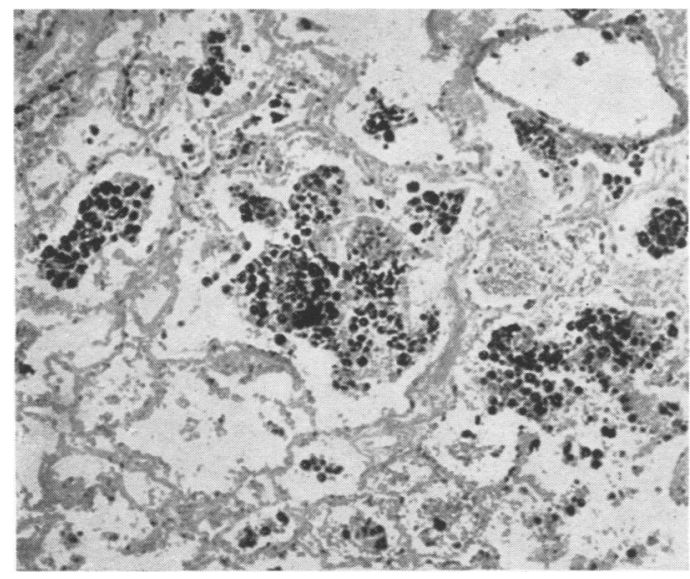

Fig. 1.-Lung alveoli containing haemosiderin-laden phagocytes. (Prussian blue, $\times$. 75.)

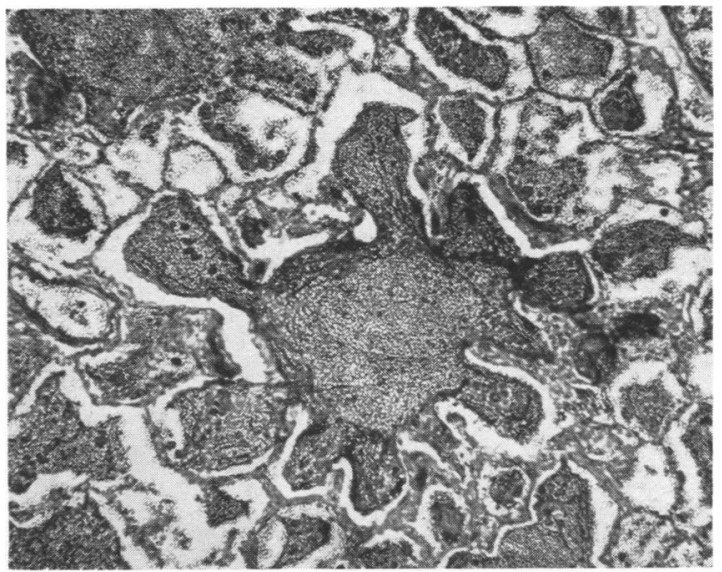

Fig. 2.-Cast in alveolar duct and related alveoli composed of red blood cells, fibrin threads, and haemosiderin-laden phagocytes. (Phosphotungstic acid haematoxylin, $\times 75$.)

limits. The small and medium-sized vessels were normal. The broncho-pulmonary and tracheobronchial lymph nodes showed simple hyperplasia and contained a small number of siderophages in the peripheral sinuses. Sections of spleen, bone marrow, and small and large intestine were normal and showed no haemosiderin.

\section{Discussion}

There are several points of interest about this case. In the first instance it is only the second case reported in an individual over 16 years of age. The reason for the higher incidence in children is unknown.

In children the condition is marked by anaemia, and although clinical signs in the chest are usually absent, radiological appearances are definite. These often superficially resemble miliary tuberculosis. Diffuse mottling is usually present, especially at the hilar areas.

In this case there was no anaemia until seven months after the onset and then it was only of mild degree. In the final stages anaemia was profound.

The episodic nature of the condition in children is similar to the repeated bouts of haemoptysis in this case. The complete absence of radiological signs in the chest is interesting. In children the radiological signs wax and wane with the severity of the attacks and with the resulting anaemia. In this case the degree of bleeding into the lung tissues must have been small until the final attack. This is mirrored by the absence of any marked anaemia until late in the disease. In the last episode, with clinical signs present in the chest, it is almost certain that radiological features would have been 
present had radiography been possible. Ellman and Gee (1951), however, suggest that haemorrhage alone does not account for the degree of anaemia in these cases, for this has been marked in cases with minimal radiological signs.

At no time was there any evidence of right heart failure, which frequently supervenes in children. Diagnosis is sometimes helped by examination of the sputum, or material obtained from lung puncture, for " heart failure cells."

Lendrum, Scott, and Park (1950) found histological changes in pulmonary haemosiderosis secondary to mitral stenosis identical with those described in the idiopathic disease. The only difference is that these changes are more widespread throughout the lung in idiopathic pulmonary haemosiderosis. The elastic tissue of the alveoli and pulmonary vessels was regarded as being within normal limits in this case, as in the case described by Nancekievill (1949).

It seems likely, therefore, that the changes in the alveolar walls and pulmonary vessels in idiopathic pulmonary haemosiderosis are secondary to haemorrhage into the lung parenchyma and not the primary cause of the haemorrhage.

We are unable to state whether the source of haemorrhage was the pulmonary capillaries or the broncho-pulmonary anastomosis. The cause of idiopathic pulmonary haemosiderosis remains unexplained.

\section{SUMMARY}

A case of idiopathic pulmonary haemosideros is described, the second reported in an adult ove the age of 16 .

The clinical picture was essentially that of recue rent haemoptysis of unknown origin.

At necropsy the lungs were enlarged, vol $\overrightarrow{\mathrm{H}}$ minous, and on section showed brown induration

Microscopically, the alveoli contained red bloo cells and haemosiderin-laden phagocytes. This elastic tissue of the alveoli and pulmonary vesseds showed no significant change.

The source of haemorrhage could not be deteg mined.

We are indebted to Dr. D. H. Collins, O.B.E., f the photomicrographs and $\mathrm{Mr}$. M. Senior for techn cal assistance.

\section{REFERENCES}

Ceelen, W. (1931). In Henke, F., and Lubarsch, O., Handbuch भु speziellen Pathologischen Anatomie und Histologie, vol. 3, pt.3 p. 20. Berlin.

Ellman, P., and Gee, A. (1951). Brit. med. J., 2, 384.

Lendrum, A. C., Scott, L. D. W., and Park, S. D. S. (1950) J. Med., 19, 249.

Nancekievill, L. (1949). Brit. med. J., 1, 431.

Scheidegger, S., and Dreyfus, A. (1945). Ann. paediat., Basel, 165 Walton, M., and Williams, A. A. (1951). Brit. med. J., 2, 390. Wyllie, W. G., Sheldon, W., Bodian, M., and Barlow, A. (194) Quart.J. Med., 17, 25. 